\title{
Analysing the Details of IoT Testing (Methodology, Framework, Challenges and Tools)
}

\author{
Rahul Sharma \\ Secretary, \\ Auricle Global Society of Education and Research, India \\ rahulmnu1@gmail.com
}

Abstract: - Internet of things is the technology used in many business organisations these days. This paper will describe the meaning of IoT and also its testing techniques. It is very important to test whatever technology is implemented in the business. Without that nobody can achieve efficiency. There is numerous testing methodology used for testing IoT technology and the devices related to it. For all type of testing techniques, there are number of challenges faced by the QA engineer, so is the case with testing of IOT devices also. This paper will also give brief description of the challenges and tools used for testing of internet of things.

Keywords: Internet of Things, Methodology, Challenges, Tools used in testing of IoT.

Introduction: - The technology which helps to provide communication between various things (devices) with wireless internet is called internet of things. With the help of IoT technology, we can operate many electronic devices with the help of internet which can be operated wirelessly through our mobile. For example, if we connect our mobile to internet and if our fridge is smart device then it can be operated with the help of our mobile phone which has internet connection. This makes the task easy and also saves a lot of time. This is the latest technology which is in trend these days.

Following are the main components of Internet of things:

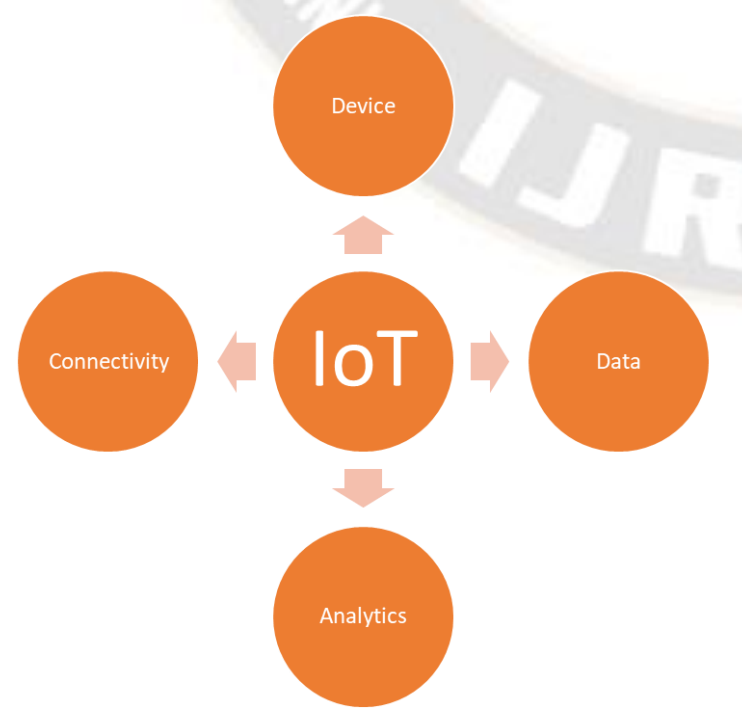

Figure1. Components of internet of things a. Device: - The device component of IoT means anything which we can to operate with implementation of IoT. There can be number of devices that can be operated after connecting them on the internet and also on the mobile phones. These days various businesses are using the concept of internet of things as it is very convenient to implement them. If the business wants to operate a lot of devices using the technique then the bandwidth of the wireless connection should be capable enough to provide uninterrupted internet connection. If the business is using many devices but the connection of wireless is poor, then there will be no point in implementing the concept of internet of things.

b. Data: - When there is communication between any number of devices, then there is definitely a large amount of data to be shared. A large amount of data is shared and hence there is facility of security also in the concept of internet of things. Therefore, IoT will collect immense amount of data from various sources in order to improve the working efficiency of the businesses. Again, the wireless internet connection should be capable of handling such large amount of data.

c. Analytics: - One of the major advantages of implementing the concept of internet of things is that it also provides the facility to analyse the data being collected. It will allow to access and share data among various devices and then also helps to analyse the data and rejects unimportant data being shared. It will gather the information from all the 
available sources, analyse it and then converts it in the language which can be easily understood by all.

d. Connectivity: - This is the most important component of the internet of things. For implementation of IoT, this is the first thing which should be proper as, everything will be connected using the wireless internet. This component basically connects all the first three components of the internet of things. If the connection is poor then there will be no sharing or gathering of data, there will not be any analysis done and hence the whole concept of IoT will not be implemented. The connection should be such that it has the capacity to handle large amount of data and also the facility to communicate within many devices without any interruption.

Hence, the concept of IoT is latest and also has many advantages. The basic requirement of Io $\mathrm{T}$ is to have efficient connectivity so that it can handle the load at any point of time as well as has the capacity to gather information from various resources and then analyse it. If the large business is implementing IoT then first of all they should make sure that the kind of connectivity which they will use should have capability to provide uninterrupted connectivity to handle large amount of data. Along with the advantages of IoT, there are also security threats. So, it is also very important to test the implementation of concept of internet of things. There are many methodologies used for testing IoT technology. Like any ither testing life cycle, there are certain stages which should be followed in order to test the threat to the devices if any.

Life Cycle of Internet of Things: - There are four main stages of the life cycle of internet of things.[1] They are

1. Design

2. Implementing

3. Maintenance

4. Decommission

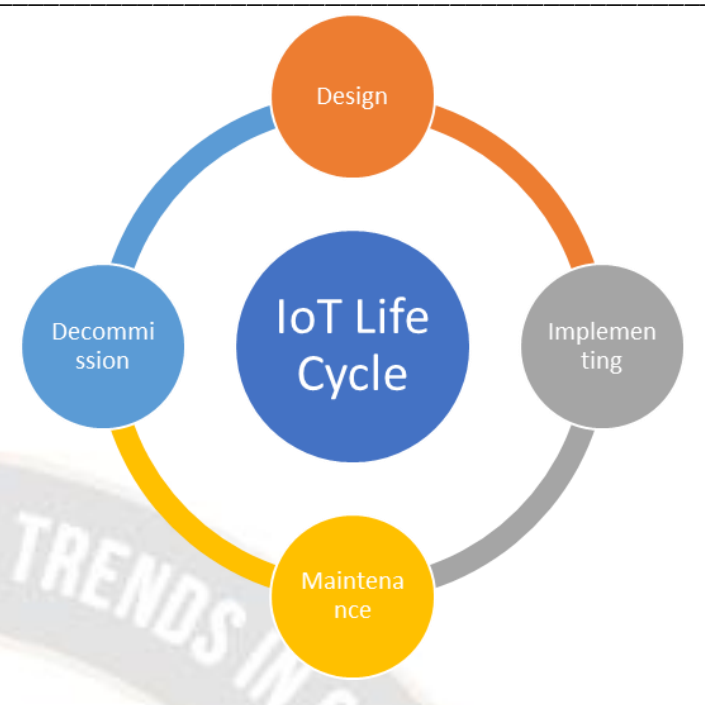

Figure 2. IoT Life Cycle

1. Design: - Like any other design phase of products, the design phase of IoT is also important as it lay foundation of the other three stages of the life cycle. First of all, the developer will understand the requirement of the business and will try to understand the technologies which the business specialize in. Then based upon these specifications, the developer will identify the scope of implementation of IoT. He will decide the number of devices which will used to implement the technology. He will also decide the wireless connection to be used. How the devices will communicate and also will determine the framework the hardware and software requirements of the system. In some cases, in order to cope up with the market needs, the business will also try to buy the already implemented IoT to use it in their own business but that also will have different set of challenges.

2. Implementing: - This is the main stage of the life cycle, as in this stage the actual configuration will take place. The Devices are connected to each other and then to the internet the to the operating device. The designer makes sure that all the planned designing will be implemented properly in this phase. Once it is done, the testers will also test whether it is being configured or not.

3. Maintenance: - This stage also known as management stage is the difficult stage of the life cycle as once the implementation is done then it is the responsibility of the developer to monitor whether the configured devices are communication properly with each other or not. They need to continuously monitor the performance of the implemented technology and it is difficult to analyse the performance without sending someone to the site. They need to monitor whether there is proper exchange of information, is the gathering of information is up to the mark, also how the data is being analysed. 
4. Decommission: - This means the last stage which includes the end of life of a device. This is also crucial stage as it is difficult to plan in the design stage the end life of any product or device. But this should be done as any device can work efficiently only up to certain period of time. Some organisations are smart enough to provide such a deal that the customers themselves will come for the change of the device. They will provide many better options and makes easy for the transition from device to another device.

IoT Testing Methodology: - [2]

Once the implementation of IoT is done, it is also very important to test the components of IoT. This will make sure that the aim of implementing IoT is met. It is essential to test the devices, connectivity and analysis of data of IoT to make sure that everything is working properly and there are no issues. Among many testing techniques, few are used to test internet of things. Following are the testing methodologies used in IoT: -

a. Security testing: - Since many devices are connected via wireless connection, it is very important that their security should be proper. There are many security threats online, so the tester needs to make sure to think of every security issue which might occur in the future. They need to think the authorisation process, encryption and decryption of data being exchanged. All the testing should be done thoroughly at the protocol level. Sometimes even if encrypted messages are sent, then also few hackers can hack it and manipulate the data. Hence, it is very important that the testers test all the possible security threats which might occur in the framework.

b. Performance Testing: - As the name says, the performance testing is related to the overall working of the technology which is implemented. This check various parameters like how the system will behave under load conditions, when data is more, when the data accessed is less. Basically, it is supposed to test the working efficiency of the system under extreme as well as minimum load conditions. In case of IoT the testing should be done at the gateway level and at the network level.

c. Compatibility testing: - It is necessary to test the compatibility of the hardware devices, software devices, protocols used, devices used, mobile compatibility, operating system used etc. This all should be done well in advance at the early stage of implementing the IoT so as to avoid future issues and errors. d. Usability application testing: - This is related to the user experience of the technology. This is to test the functionality of the IoT> This is to make sure that the devices are working as per expectation or not. Whether each function of the IoT is being done properly or not.

e. Data Connectivity testing: - This type of testing is very important part of testing of internet of things as it will check the connection between many devices, protocols used, etc. It makes sure that the devices are communicating without any interruption, and, they are able to handle the load in case of more devices used.

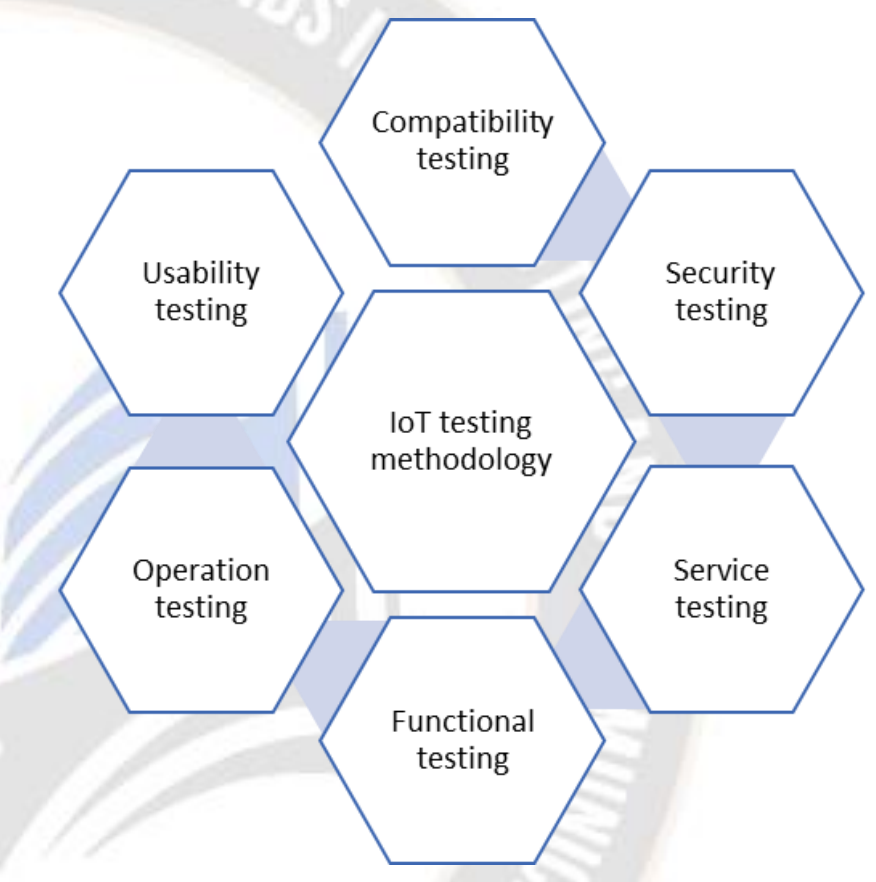

Figure4 IoT testing Techniques

The above-mentioned types of testing are common types of testing methodologies used in IoT. But in some cases, the testers should determine scope of testing of the IoT. Hence, the testers testing the IoT technology should have idea of implementation of the technique, only then they will be able to identify all possible types of testing which is important to do. 


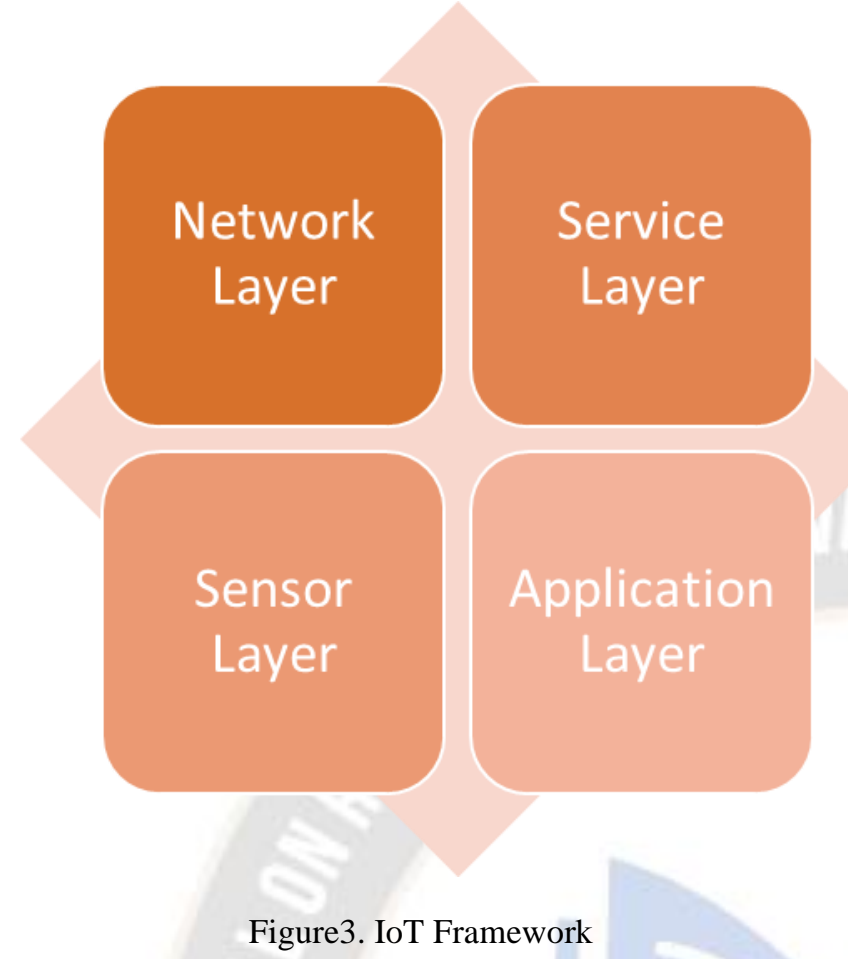

Testing conducted across different layers of IoT: -

There are four layers of Internet of Things: -

- Application Layer: The IoT is implemented here in this layer and hence it is important to conduct following testing at this level: -

a. Functional Testing

b. Compatibility testing

c. User Testing

d. API testing

b. Service Layer: - The types of services for which the concept of IoT is implemented is visible in this phase. Following are the testing types conducted in this phase: -

a. Connection testing

b. Functional testing

c. API testing

c. Network Layer: - With the help of this layer, the devices are connected to each other and can communicate with each other. So, two types of testing which is Network compatibility and Network connectivity are important to conduct at this layer.

d. Sensor Layer: - Functional testing and security testing are important to conduct at this layer if IoT. Whenever data is exchanged online, there are several security threats issues which should be considered and then testing should be planned. All the devices should be test of they might have some security issues.
Advantages of implementing IoT: -

There are many advantages of having internet of thigs technology: -

1.Saves Time: - This is the main advantage of the IoT as it will have all the devices connected to one place and those can be operated using wireless connection without even going there, means no human involvement and so this in turn saves a lot of time and energy.

2. Satisfied Customers: - This technology benefits customers as well as it gives them opportunity to operate many things at the same time. This in turn will improve the customer business relationship. The customer will have more projects which they want to implement using the technology as it saves a lot of time. Hence, the business and organisation which provides the opportunity to implement IoT will attract more users.

3. Improved efficiency of business: -The businesses which provides the facility of implementing the IoT will be able to enhance their efficiencies as this technology gives options to use many devices at the same time using wireless connection

4. Gives new opportunities: - As the IoT also helps to analyse the data and also gives the option to gather data from various sources, it also gives new business opportunities. This in turn will make the business successful.

5. Helps to identify the issues in advance: - The technology also provides the ability to predict the issues that might occur in future as the collected data can be analysed. This way the issues can also be prevented before they arise.

6. More Revenue generation: - As it is the latest technology used, everybody wants to save their time and energy. If it is implemented properly then it can also help to generate more revenue which in turn will give more options to invest in more IoT technology and the business will flourish in future.

Disadvantages of IoT: -[3]

1. Complex nest: - The process of implementation of IoT is very complex. It needs better understanding of all the components used in the IoT implementation process. The designer who is responsible for designing the process of implementation of IoT should have sound knowledge of the process otherwise it will not give favourable results.

2. Security: - Although, the testers checks all the possible threats which can cause security issues, then also in some situations It is difficult to identify the security and network threats.

3. Difficult to find the affected device: - In IoT many devices are used. The more the devices the more it 
gets difficult to manage these devices. Even if someone's device is hacked, they will not come to know about is as there are many layers and devices involved in the technology.

4. Home security issues: - These days due to many advantages of IoT, many offices and even in houses, this technology is in use. But if not everybody knows the best protocols to follow in order to make sure the security parameter is met, there is more threat to people. Even if small amount of information is leaked the hackers will take advantage of it.

5. Not changing the default admin details: - Some people does not change the default user login details as a result of which the hackers can attack the system and can access confidential information. The manufacturers also sometimes never ask the customers to change the default credentials. So, in such cases there is threat to the devices and the information stored in the device.

\section{Tools used for IoT: - [4]}

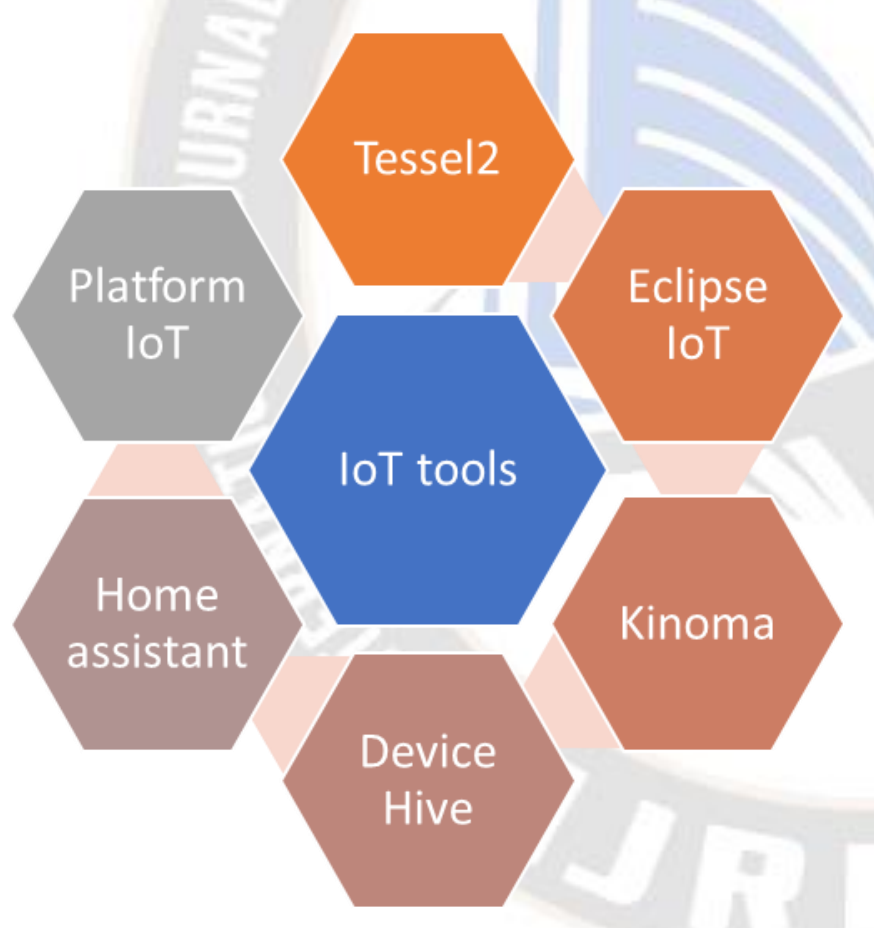

Figure 4 Internet of Things Tools c. Kinoma: - It is semiconductor hardware platform which is divided in to two parts. One is called Kinoma create which is used to implement IoT devices and the other is element board which is used for IoT product platform.

d. Device Hive: - It is the most commonly used tool for IoT implementation. This tool provides the facility of implementing IoT for smart home devices.

e. Home Assistant: - This tool is used for smart home devices. This supports python language. With the help of this it is very easy to implement devices using IoT in home. This also make sure that it is safe and there are no threats using this.

f. Platform IoT: - This tool comes with integrated debugger. This is useful for mobile app development and also gives the opportunity to implement IoT using mobile.

Conclusion: - In this paper it is discussed that how useful is inter of things technology. It has many advantages and also few disadvantages. If implemented effectively it can do wonders for the customers as well as the business. It is very important to test the implemented technology otherwise it will not give expected results. The testing techniques used will depend upon the type of devices used, the type of connection used and many other factors. Hence, the technology helps the business to save time and also improve the efficiency.

\section{References: -}

1.https://www.avsystem.com/blog/iot-device-lifecyclemanagement/

2.https://www.clariontech.com/blog/iot-testing-framework 3.https://readwrite.com/2019/09/05/9-

4.https://www.educba.com/iot-tools/

a. Tessel 2: - This is the basic tool which can be used to make simple IoT prototypes and applications. This provides the facility of many sensors and modules with the help of which the concept of IoT can easily be implemented.

b. Eclipse IoT: - This tool helps to implement many IoT projects which includes IoT framework, IoT protocols etc. This also supports Lua programming language which is used for IoT. 